九州大学学術情報リポジトリ

Kyushu University Institutional Repository

\title{
A GRAMMATICAL INFERENCE FOR CONTEXT-FREE LANGUAGES BASED ON SELF-EMBEDDING
}

Tanatsugu, Keisuke

Department of Mathematics, Kitakyushu University

https://doi.org/10.5109/13382

出版情報: Bulletin of informatics and cybernetics. 22 (3/4), pp.149-163，1987-03. Research Association of Statistical Sciences

バージョン:

権利関係 : 


\title{
A GRAMMATICAL INFERENCE FOR CONTEXT-FREE LANGUAGES BASED ON SELF-EMBEDDING
}

\author{
By
}

\author{
Keisuke Tanatsugu*
}

\begin{abstract}
Our purpose in this paper is to construct a practical algorithm to inductively infer context-free languages. A basic procedure is first introduced to take out the self-embedding structure from given strings. Secondly, based on this procedure an algorithm to infer linear grammars from given finite sample sets is presented and its completeness is proved. Finally, by using a method to compose linear grammars, we propose an algorithm which is also complete for context-free languages.
\end{abstract}

\section{Introduction}

For a language $L$ over an alphabet $\Sigma$, a finite subset of $I(L)=\{+w ; w \in L\} \cup$ $\left\{-w ; w \in \Sigma^{*}-L\right\}$ is said a sample of $L$. Given a family of languages $\mathcal{L}$, an algorithm $f$ is called a complete grammatical inference algorithm for $\mathcal{L}$ if for any $L \in \mathcal{L}$ there exist some infinite sequence $x_{1}, x_{2}, \cdots \in I(L)$ and integer $n_{0}$ such that $n \geqq n_{0}$ implies $L=L$ $\left[f\left(x_{1}, x_{2}, \cdots, x_{n}\right)\right]$. We may find such an algorithm by enumerating all grammars even for the whole of phrase structure languages [3]. However, we concern with an effective algorithm which enumerates only qualified grammars using structural information belonging to samples. Biermann [5] introduced such an interesting algorithm for regular languages and Tanatsugu [7] proposed for harmonic linear languages which is a superclass of regular languages. These method are based on the fact that, each variable in grammar to be inferred, may be characterized by a finite subset of the language generated from it.

In the present paper we construct an practical inference procedure for the family of context-free languages. We consider that the main structural feature of context-free languages is the self-embedding. So, we first give the procedure to take out redunduncy parts in both sides of strings by using the notion of derivative mapping [2], in section 3 . Based on this procedure, we present an inference algorithm for linear languages. Then it is shown that this algorithm is complete for the family of linear languages in section 4. Futhermore, by adding a composition method of linear grammars, we propose an algorithm which is also complete for the family of context-free languages in section 5.

\footnotetext{
* Department of mathematics, Kitakyushu University, Kitakyushu 802, Japan.
} 


\section{Preliminaries}

We shall give some basic notions and lemmas for the subsequent sections.

Let $X$ be an alphabet, then the set of all strings over $X$ is denoted by $X^{*}$. The empty string of zero symbols is denoted by $\varepsilon$.

A context-free grammar (CFG for short) is defined by a quadruple $G=(\Gamma, \Sigma, P, S$ ) where $\Gamma$ is a finite set of variable symbols, $\Sigma$ called an alphabet is a finite set of terminal symbols, $P$ is a finite set of production rules of the form $A \rightarrow x$ with $A \in \Gamma$ and $x \in(\Gamma \cup \Sigma)^{*}$, and $S \in \Gamma$ is the start symbol. We write $x \Rightarrow y$ if there exist $A \in \Gamma$, $x_{1}, x_{2}, z \in(\Gamma \cup \Sigma)^{*}$ such that $x=x_{1} A x_{2}, y=x_{1} z x_{2}$ and the rule $A \rightarrow z$ is in $P$. The transitive closure of $\Rightarrow$ is written $\stackrel{*}{\Rightarrow}$. The language $L_{A}$ generated from $A \in \Gamma$ is denoted by $\left\{w ; A \stackrel{*}{\Rightarrow} w, w \in \Sigma^{*}\right\}$. Especially $L_{S}$ is also represented as $L(G)$ and it is said the context-free language (CFL for short) generated by the grammar $G$. Now we add the following restrictions for every $\mathrm{CFG} G=(\Gamma, \Sigma, P, S)$ :

1. For each variable $A \in \Gamma$ :

(1) There is a derivation $S \stackrel{*}{\Rightarrow} u A v$ for some $u, v \in(\Gamma \cup \Sigma)^{*}$.

(2) $L_{A} \neq \phi$.

(3) $(A \rightarrow A) \notin P$.

(4) If $A \neq S$, then there is at least one production rule of the form $A \rightarrow \alpha A \beta$ where $\alpha, \beta \in(\Gamma \cup \Sigma)^{*}$.

2. $L_{A} \neq L_{B}$ for any $A, B \in \Gamma$ such that $A \neq B$.

In spite of the above restrictions, for any $\mathrm{CFL} L \subset \Sigma^{*}$ there exists a grammar $G$ such that $L=L(G)$. A CFG $G$ is called linear if every production rule is the form of $A \rightarrow u B v$ or $A \rightarrow w$ with $A, B \in \Gamma$ and $u, v, w \in \Sigma^{*}$, then $L(G)$ is said a linear language.

Definition 1. Let $L$ be a language over $\Sigma$, then we denote a derivative language of $L$ by

$$
\bar{u} L \bar{v}=\{x ; u x v \in L\} .
$$

Then $(u, v)$ is called a cover of $L$ for $\bar{u} L \bar{v}$. Futhermore, $L$ is said to be embedding with respect to $(u, v)$ if $L$ is a subset of $\bar{u} L \bar{v}$.

The following is immediately obtained from the above definition.

LEMMA 1. 1. Let $L_{1}, L_{2}, L$ be languages over $\Sigma$ and $u, u_{1}, u_{2}, v, v_{1}, v_{2} \in \Sigma^{*}$, then the following relations hold:

(1) $\bar{u}_{2}\left(\bar{u}_{1} L \bar{v}_{1}\right) \bar{v}_{2}=\overline{u_{1} u_{2}} L \overline{v_{2} v_{1}}$.

(2) If $L_{1} \subset L_{2}$, then $\bar{u} L_{1} \bar{v} \subset \vec{u} L_{2} \bar{v}$.

(3) $\bar{u}\left(L_{1} \cup L_{2}\right) \bar{v}=\bar{u} L_{1} \bar{v} \cup \bar{u} L_{2} \bar{v}$.

(4) $\bar{u}\left(L_{1} \cap L_{2}\right) \bar{v}=\bar{u} L_{1} \bar{v} \cap \bar{u} L_{2} \bar{v}$.

2. Let $G=(\Gamma, \Sigma, P, S)$ be a $C F G$. If $A \stackrel{*}{\Rightarrow} u B v$ then $L_{B} \subset \bar{u} L_{A} \bar{v}$ where $A, B \in \Gamma$ and $u, v \in \Sigma^{*}$.

We now introduce an order relation on $\Sigma^{*} \times \Sigma^{*}$ based on the lexicographical order on $\Sigma^{*}$ as follows :

if and only if

$$
\left(u_{1}, v_{1}\right)<\left(u_{2}, v_{2}\right)
$$




$$
\left(\left|u_{1} v_{1}\right|<\left|u_{2} v_{2}\right|\right) \vee\left(\left|u_{1} v_{1}\right|=\left|u_{2} v_{2}\right| \wedge u_{1} v_{1}<u_{2} v_{2}\right) \vee\left(u_{1} v_{1}=u_{2} v_{2} \wedge v_{1}<v_{2}\right)
$$

For example, for $\Sigma=\{a, b\}$

$(\varepsilon, \varepsilon)<(a, \varepsilon)<(\varepsilon, a)<(b, \varepsilon)<(\varepsilon, b)<(a a, \varepsilon)<(a, a)<(\varepsilon, a a)<(a b, \varepsilon)<(a, b)<\cdots$.

We represent the successor of $(u, v)$ as $(u, v)^{\prime}$.

Definition 2. Let $X, Y \subset \Sigma^{*}$ and $\alpha, \beta \in \Sigma^{*}$.

(1) $\mathcal{E}_{X}(\alpha, \beta)=\left\{X^{\prime} \neq \phi ; X^{\prime} \subset \bar{\alpha} X^{\prime} \bar{\beta}, X^{\prime} \subset X\right\}$.

(2) $M_{X}(\alpha, \beta)=\cup \mathcal{E}_{X}(\alpha, \beta)$.

(3) $\mathcal{E}_{X}=\left\{M_{X}(\alpha, \beta) ;(\alpha, \beta) \neq(\varepsilon, \varepsilon)\right\}$.

(4) $\hat{M}_{X ; Y}(\alpha, \beta)=X \cap M_{Y}(\alpha, \beta)$. We simply write $\hat{M}_{X}(\alpha, \beta)$ in the case that $Y$ is evident.

The following facts are easily derived from the above definition:

Lemma 2. For any $X \in \Sigma^{*}$ and $\alpha, \beta \in \Sigma^{*}$ :

(1) $M_{X}(\alpha, \beta) \subset X$. Particularly, $M_{X}(\varepsilon, \varepsilon)=X$.

(2) $M_{X}(\alpha, \beta) \subset \bar{\alpha} M_{X}(\alpha, \beta) \bar{\beta}$.

(3) $\mathcal{E}_{X}=\phi$ if $X$ is finite.

EXAMPLE 1. Let $X=\left\{a^{m} b^{m} ; m \geqq 0\right\} \cup\left\{a^{n} b^{2 n} ; n \geqq 0\right\}$, then

$$
\begin{aligned}
& \mathcal{E}_{X}(a, b)=\left\{\left\{a^{m} b^{m} ; m \geqq 0\right\},\left\{a^{m} b^{m} ; m \geqq 1\right\}, \cdots\right\}, \\
& \mathcal{E}_{X}(a, b b)=\left\{\left\{a^{n} b^{2 n} ; n \geqq 0\right\},\left\{a^{n} b^{2 n} ; n \geqq 1\right\}, \cdots\right\}, \\
& M_{X}(a, b)=M_{X}\left(a^{2}, b^{2}\right)=\cdots=\left\{a^{m} b^{m} ; m \geqq 0\right\}, \\
& M_{X}(a, b b)=M_{X}\left(a^{2}, b^{4}\right)=\cdots=\left\{a^{n} b^{2 n} ; n \geqq 0\right\}, \\
& \mathcal{E}_{X}=\left\{\left\{a^{m} b^{m} ; m \geqq 0\right\},\left\{a^{n} b^{2 n} ; n \geqq 0\right\}\right\} .
\end{aligned}
$$

Lemma 3. Let $G=(\Gamma, \Sigma, P, S)$ be $a C F G$ and $A \in \Gamma$. If $S \stackrel{*}{\Rightarrow} u A v$ and $A \stackrel{*}{\Rightarrow} \alpha A \beta$, then

$$
L_{A} \subset M_{\bar{u} L(G) \bar{v}}(\alpha, \beta)
$$

where $(u, v),(\alpha, \beta) \in \Sigma^{*} \times \Sigma^{*}$.

Proof. By Lemma 1, $L_{A} \subset \bar{u} L(G) \bar{v}, L_{A} \subset \bar{\alpha} L_{A} \bar{\beta}$. Hence

Since

$$
L_{A} \in \mathcal{E}_{\bar{u} L(G) \bar{v}}(\alpha, \beta) .
$$

we obtain

$$
M_{\bar{u} L(G) \bar{v}}(\alpha, \beta)=\cup \mathcal{E}_{\bar{u} L(G) \bar{v}}(\alpha, \beta),
$$

$$
L_{A} \subset M_{\bar{u} L(G) \bar{v}}(\alpha, \beta) .
$$

Lemma 4. Let $G=(\Gamma, \Sigma, P, S)$ be a $C F G$ and $L=L(G)$. If

then

$$
S \stackrel{*}{\Rightarrow} u A v, \quad A \stackrel{*}{\Rightarrow} u^{\prime} B v^{\prime}, \quad A \stackrel{*}{\Rightarrow} \alpha A \beta \quad \text { and } \quad B \stackrel{*}{\Rightarrow} \alpha^{\prime} B \beta^{\prime},
$$

$$
L_{B} \subset \bar{u}^{\prime} M_{\bar{u} L \bar{v}}(\alpha, \beta) \bar{v}^{\prime} \cap M_{\overline{u u^{\prime}} L \overline{v^{\prime} v}}\left(\alpha^{\prime}, \beta^{\prime}\right)
$$

where $(u, v),\left(u^{\prime}, v^{\prime}\right),(\alpha, \beta),\left(\alpha^{\prime}, \beta^{\prime}\right) \in \Sigma^{*} \times \Sigma^{*}$.

Proof. By Lemma 3

$$
L_{A} \subset M_{\bar{u} L \bar{v}}(\alpha, \beta), \quad L_{B} \subset M_{\overline{u u^{\prime}} L \overline{v^{\prime} v}}\left(\alpha^{\prime}, \beta^{\prime}\right) .
$$


And $L_{B} \subset \bar{u}^{\prime} L_{A} \bar{v}^{\prime}$ since $A \stackrel{*}{\Rightarrow} u^{\prime} B v^{\prime}$. Therefore,

$$
\bar{u}^{\prime} M_{\bar{u} L \bar{v}}(\alpha, \beta) \bar{v}^{\prime} \cap M_{\overline{u u^{\prime}} L \overline{v^{v} v}}\left(\alpha^{\prime}, \beta^{\prime}\right) \supset \bar{u}^{\prime} L_{A} \bar{v}^{\prime} \cap L_{B}=L_{B} .
$$

We now denote $X_{k}(\alpha, \beta)$ by $\bigcap_{i=0}^{k} \bar{\alpha}^{i} X \bar{\beta}^{i}$ where $X \subset \Sigma^{*}, \alpha, \beta \in \Sigma^{*}$, accordingly, $X_{\infty}(\alpha, \beta)$ indicates $\bigcap_{i=0}^{\infty} \bar{\alpha}^{i} X \bar{\beta}^{i}$.

Lemma 5. If $X \subset \bar{\alpha} X \bar{\beta}$, then $M_{X}(\alpha, \beta)=X_{\infty}(\alpha, \beta)$ where $X \subset \Sigma^{*}, \alpha, \beta \in \Sigma^{*}$.

Proof. Let $x \in M_{X}(\alpha, \beta)$, then there exists $X^{\prime}$ such that $X^{\prime} \subset X, X^{\prime} \subset \bar{\alpha} X^{\prime} \bar{\beta}$ and $x \in X^{\prime}$. Therefore, for any integer $i$

$$
x \in X^{\prime} \subset \bar{\alpha}^{i} X^{\prime} \bar{\beta}^{i} \subset \bar{\alpha}^{i} X \bar{\beta}^{i} \quad \text { i. e., } \quad x \in X_{\infty}(\alpha, \beta) .
$$

Inversely let $x \in X_{\infty}(\alpha, \beta)$, then

that is,

$$
x \in X, \bar{\alpha} X \bar{\beta}, \bar{\alpha}^{2} X \bar{\beta}^{2}, \cdots
$$

$$
x, \alpha x \beta, \alpha^{2} x \beta^{2}, \cdots \in X .
$$

Now let $X^{\prime}=\left\{\alpha^{i} x \beta^{i} ; i \geqq 0\right\}$, then $X^{\prime} \subset X$ and $X^{\prime} \subset \bar{\alpha} X^{\prime} \bar{\beta}$, hence it follows that $x \in X^{\prime} \subset$ $M_{X}(\alpha, \beta)$.

\section{Inference Algorithm}

\subsection{Generation of Variables}

Let $L$ be a CFL and any grammar generating $L$ be unknown. Giving a sample $I$ of $L$, we shall consider the method to infer a grammar $G=(\Gamma, \Sigma, P, S)$ of $L$. We start with the specification of variables $\Gamma^{\prime}$ corresponding to $\Gamma$ in $G$. Since $S \stackrel{*}{\Rightarrow} u A v$ and $(A \rightarrow \alpha A \beta) \in P$ imyly

$$
L_{A} \subset M_{\bar{u} L \bar{v}}(\alpha, \beta) \subset \bar{u} L \bar{v},
$$

it is natural that we select a $(u, v)$ satisfying the above precondition for $A \in \Gamma$ and regard $M_{\bar{u} L \bar{v}}(\alpha, \beta)$ as the corresponding variable to $A$. However, since $M_{\bar{u} L \bar{v}}(\alpha, \beta)$ is generally infinite set, we take the following finite set instead of it as a variable in $\Gamma$ :

$$
\hat{M}_{\bar{u} I_{+} \bar{v} ; \bar{u} L \bar{v}}(\alpha, \beta)=\bar{u} I_{+} \bar{v} \cap M_{\bar{u} L \bar{v}}(\alpha, \beta)
$$

So, first, we shall show an algorithm to construct the finite set $X \cap W_{k}(\alpha, \beta)\left(X \subset W \subset \Sigma^{*}\right)$ by Procedure DER in Fig. 1.

Notice that the operation $Y \cap \bar{\alpha}^{k} W \bar{\beta}^{k}$ in Procedure DER may be surely excuted because $Y$ is finite even though $\bar{\alpha}^{k} W \bar{\beta}^{k}$ is infinite, under the actual assumption that we can always know whether $x \in W$ or not for every $x \in \Sigma^{*}$.

Proposition 1. Procedure DER terminates in finite steps and its final output is $Y=X \cap W_{k}(\alpha, \beta)$.

Proof. First we shall prove that $X^{\prime}=X \cap W_{k}(\alpha, \beta)$ in arbitrary step $k$.

This holds in the case of $k=0$ since $X \subset W=W_{0}(\alpha, \beta)$. Suppose that this holds in stage $k-1(k \geqq 1)$ and consider the case of stage $k$. As $Y$ in stage $k$ is $X^{\prime}$ in stage $k-1$, 


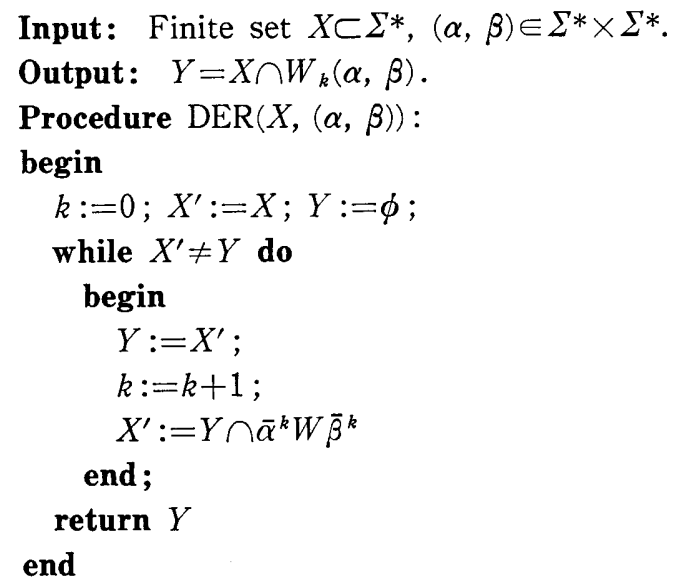

Fig. 1. Procedure DER.

$$
\begin{aligned}
X^{\prime} & =Y \cap \bar{\alpha}^{k} W \bar{\beta}^{k} \\
& =\left(X \cap W_{k-1}(\alpha, \beta)\right) \cap \bar{\alpha}^{k} W \bar{\beta}^{k} \\
& =X \cap W_{k}(\alpha, \beta)
\end{aligned}
$$

Therefore $X^{\prime}=X \cap W_{k}(\alpha, \beta)$ for any stage $k$.

Thus if $X^{\prime}=Y$ in stage $k$, then Procedure DER stops and $Y=X \cap W_{k}(\alpha, \beta)$. If $X^{\prime} \subsetneq Y$ in stage $k$, then $X^{\prime}$ get to $Y$ in the next stage. Since $Y$ strictly decreases as stage $k$ increases, $X^{\prime}=Y$ is resulted in finite steps.

Now we shall show that the output of Procedure DER coincides to $\hat{M}_{X ; W}(\alpha, \beta)$ for an appropriate input $X \subset W$. Such a $X$ is obtained by extending some finite set $X_{0} \subset W$ for a given $\left(\alpha_{0}, \beta_{0}\right) \in \Sigma^{*} \times \Sigma^{*}$ as follows :

Procedure EXT.

Step 1. $X \leftarrow X_{0}$.

Step 2. $(\alpha, \beta) \leftarrow(\varepsilon, \varepsilon)$.

Step 3. $(\alpha, \beta) \leftarrow(\alpha, \beta)^{\prime}, k \leftarrow 1$.

Step 4. If $X \cap W_{k-1}(\alpha, \beta) \subset M_{W}(\alpha, \beta)$, then go to step 7 .

Step 5. If $X \cap W_{k-1}(\alpha, \beta) \neq X \cap W_{k}(\alpha, \beta)$, then add 1 to $k$ and go to step 4 .

Step 6. There is an integer $p$ such that $x, \alpha x \beta, \cdots \alpha^{p} \beta^{p} \in W$ and $\alpha^{p+1} x \beta^{p+1} \notin W$ for each member $x$ of $X \cap W_{k-1}(\alpha, \beta)-M_{W}(\alpha, \beta)$. Let $p_{0}$ be the minimal integer of them and $x_{0}$ designate one of the strings corresponding to the intger $p_{0}$. Add $\left\{\alpha x_{0} \beta, \cdots, \alpha^{p_{0}-k+1} x_{0} \beta^{p_{0}-k+1}\right\}$ to $X$, and go to step 2 .

Step 7. If $(\alpha, \beta)<\left(\alpha_{0}, \beta_{0}\right)$ then go to step 3 .

Step 8. Stop.

We have the below propositions related to Procedure EXT.

Proposition 2. Integer $p_{0}$ is always well defined in step 6 and $p_{0} \geqq k$.

PROOF. First we show that there exists certainly integer $p_{0}$ in step 6 . If there is no integer $p$ such that $x, \alpha x \beta, \cdots, \alpha^{p} x \beta^{p} \in W$ and $\alpha^{p+1} x \beta^{p+1} \notin W$, then, since $x \in W$, $\left\{\alpha^{n} x \beta^{n} ; n \geqq 0\right\}$ is a subset of $W$, i. e., of $M_{W}(\alpha, \beta)$. This contradicts $x \notin M_{W}(\alpha, \beta)$.

Secondly we show that $p_{0} \geqq k$. If $x \in X \cap W_{k-1}(\alpha, \beta)$, then $p_{0} \geqq k-1$ because 
$x, \alpha x \beta, \cdots, \alpha^{k-1} x \beta^{k-1} \in W$. In the case of $p_{0}=k-1, x_{0}$ is in $X \cap W_{k-1}(\alpha, \beta)$ but not $W_{k}(\alpha, \beta)$, in other words,

$$
X \cap W_{k-1}(\alpha, \beta) \neq X \cap W_{k}(\alpha, \beta) .
$$

This derives a contradiction. Thus $p_{0}>k-1$.

Proposition 3. Procedure EXT stops in finite steps.

PROOF. Notice that for any $(\alpha, \beta)$ there is $k$ such that $X \cap W_{k-1}(\alpha, \beta)=X \cap W_{k}(\alpha, \beta)$. Let us set $k, X$ to $k_{1}, X_{1}$, respectively, at the time that step 6 has been excuted for $(\alpha, \beta)$, and set $k, X$ to $k_{2}, X_{2}$, respectively, at some later time that step 6 has been again excuted for $(\alpha, \beta)$. Then we shall show that $k_{1}<k_{2}$.

and

$$
\begin{array}{ll}
X_{1} \cap W_{k-1}(\alpha, \beta) \not \subset M_{W}(\alpha, \beta) & \left(k \leqq k_{1}\right), \\
X_{1} \cap W_{k-1}(\alpha, \beta) \neq X_{1} \cap W_{k}(\alpha, \beta) & \left(k<k_{1}\right)
\end{array}
$$

Since $X_{1} \subset X_{2}$,

$$
X_{1} \cap W_{k-1}(\alpha, \beta)=X_{1} \cap W_{k}(\alpha, \beta) \quad\left(k=k_{1}\right)
$$

$$
X_{2} \cap W_{k-1}(\alpha, \beta) \not \subset M_{W}(\alpha, \beta) \quad\left(k \leqq k_{1}\right) .
$$

Since $X_{1} \subset X_{2}$ and $W_{k-1}(\alpha, \beta) \supset W_{k}(\alpha, \beta)$,

$$
X_{2} \cap W_{k-1}(\alpha, \beta) \neq X_{2} \cap W_{k}(\alpha, \beta) \quad\left(k \leqq k_{1}\right) .
$$

When $k=k_{1}$, since

$$
\begin{aligned}
& \alpha^{p_{0}-k+1} x_{0} \beta^{p_{0}-k+1} \in X_{2}, \quad \alpha^{p_{0}} x_{0} \beta^{p_{0}} \in W, \quad \alpha^{p_{0}+1} x_{0} \beta^{p_{0}+1} \notin W, \\
& X_{2} \cap W_{k-1}(\alpha, \beta) \ni \alpha^{p_{0}-k+1} x_{0} \beta^{p_{0}-k+1} \notin X_{2} \cap W_{k}(\alpha, \beta) .
\end{aligned}
$$

That is,

$$
X_{2} \cap W_{k-1}(\alpha, \beta) \neq X_{2} \cap W_{k}(\alpha, \beta) \quad\left(k \leqq k_{1}\right) .
$$

Thus we obtain $k_{1}<k_{2}$.

Let us set the maximal number of $p_{0}$ 's for every $(\alpha, \beta)$ no larger than $\left(\alpha_{0}, \beta_{0}\right)$, as $p^{*}$, then $p^{*} \geqq p_{0} \geqq k$ by Proposition 3. Therefore, it turns out that the step 6 is never excuted for any $(\alpha, \beta)$ after some stage. That is, for any $(\alpha, \beta)$, an integer $k$ is found such that $X \cap W_{k-1}(\alpha, \beta) \subset M_{W}(\alpha, \beta)$. Thus Procedure EXT stops.

Theorem 1. Let $\left(\alpha_{0}, \beta_{0}\right) \in \Sigma^{*} \times \Sigma^{*}$. In Procedure DER, for any $(\alpha, \beta) \leqq\left(\alpha_{0}, \beta_{0}\right)$, there exists a finite subset $X$ of $W$ such that:

(1) If $M_{W}(\alpha, \beta)=\phi$, then the output $D E R(X,(\alpha, \beta))$ is $\phi$.

(2) If $M_{W}(\alpha, \beta) \neq \phi$, then the output $D E R(X,(\alpha, \beta))$ is nonempty set $\hat{M}_{X ; W}(\alpha, \beta)$.

PROOF. We may consider a finite subset $X_{0}$ of $W$ such that $M_{W}(\alpha, \beta) \cap X_{0} \neq \phi$ for any $(\alpha, \beta) \leqq\left(\alpha_{0}, \beta_{0}\right)$ satisfying $M_{W}(\alpha, \beta) \neq \phi$. For example, such a $X_{0}$ is easily obtained by taking out, one by one, a element from every nonempty $M_{W}(\alpha, \beta)$. Let $X$ be the set obtained extending $X_{0}$ by Procedure EXT. Then for any $(\alpha, \beta) \leqq\left(\alpha_{0}, \beta_{0}\right)$ there is an integer $k_{0}$ such that

Now if

$$
\begin{array}{lll}
X \cap W_{k-1}(\alpha, \beta) \neq X \cap W_{k}(\alpha, \beta) & \text { if } & k<k_{0}, \\
X \cap W_{k-1}(\alpha, \beta) \subset M_{W}(\alpha, \beta) & \text { if } & k=k_{0} .
\end{array}
$$


Input: $(u, v),(\alpha, \beta) \in \Sigma^{*} \times \Sigma^{*}, Y \subset \Sigma^{*}, n_{0}, r, r_{0} \in N$, linear grammar $\hat{G}=\left(\hat{\Gamma}, \Sigma, \hat{P}, A_{1}\right)$. Output: New variable $A$ if possible and all production rules related to $A$. Voriables:

$\mu(i)$ : Finite set corresponding to $i$-th variable $A_{i} \in \hat{\Gamma}$.

$\hat{\Gamma}(u, v) \subset \hat{\Gamma}$ : Set of vriables generated under $(u, v)$.

$\mathrm{SUB} \subset \Sigma^{*} \times \Sigma^{*} \times N$ : Construct the rule $A_{x} \rightarrow u^{\prime} A_{r} v^{\prime}$ if $\left(\left(u^{\prime}, v^{\prime}\right) x\right) \in \mathrm{SUB}$.

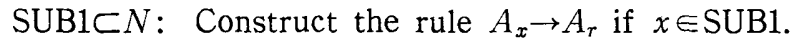

SUB2 $\subset N$ : Construct the rule $A_{r} \rightarrow A_{x}$ if $x \in \mathrm{SUB} 2$.

$r_{0}$ : Number of variable generated at first under $(u, v)$.

check: 0 if no variable is generated; 1 if one variable is generated; 2 if two variables or more is generated under $(u, v)$.

procedure $\operatorname{LG}\left(Y,(u, v),(\alpha, \beta), n_{0}, r, r_{0}, \hat{G}\right)$ :

bcgin

check $:=0$;

if $Y \neq \phi$ then

begin

if $r<r_{0}$ then check: $=1$

else if $Y \neq \mu(i)$ for all $i\left(r_{0} \leqq i \leqq r\right)$ then check:=2;

if check $\neq 0$ then

begin

SUB $:=\phi ;$ SUB1 $:=\phi ;$ SUB2 $:=\phi$;

if $r \neq 1$ then

for $i=1$ until $r_{0}-1$ do

if $Y \cap \bar{u}^{\prime} \mu(i) \bar{v}^{\prime} \neq \phi$ for some $\left(u^{\prime}, v^{\prime}\right)$ such that

$(u, v)=\left(u^{\prime \prime} u^{\prime}, v^{\prime} v^{\prime \prime}\right)$ and $A_{i} \in \hat{\Gamma}\left(u^{\prime \prime}, v^{\prime \prime}\right)$ then

$\left.\mathrm{SUB}:=\mathrm{SUB} \cup\left\{\left(u^{\prime}, v^{\prime}\right), i\right)\right\}$;

if $\mathrm{check}=2$ then

for $i=r_{0}$ until $r$ do

if $Y \subset \mu(i)$ then SUB1:=SUB1 $\cup\{i\}$

else if $Y \supset \mu(i)$ then SUB2:=SUB2 $\cup\{i\}$;

$r:=r+1$;

$\mu(r):=Y ; \hat{\Gamma}(u, v):=\hat{\Gamma}(u, v) \cup\left\{A_{r}\right\} ; \hat{\Gamma}:=\hat{\Gamma} \cup\left\{A_{r}\right\} ;$

$\hat{P}=\hat{P} \cup\left\{A_{r} \rightarrow \alpha A_{r} \beta\right\}$

$\cup\left\{A_{x} \rightarrow u^{\prime} A_{r} v^{\prime} ;\left(\left(u^{\prime}, v^{\prime}\right), x\right) \in \mathrm{SUB}\right\}$

$\cup\left\{A_{x} \rightarrow A_{r} ; x \in \mathrm{SUB} 1\right\} \cup\left\{A_{r} \rightarrow A_{x} ; x \in \mathrm{SUB} 2\right\}$

$\cup\left\{A_{r} \rightarrow w ;|w| \leqq n_{0}, w \in \mu(r)\right\}$

end

else if there is no derivation such that $A_{i} \stackrel{*}{\Rightarrow} \alpha A_{i} \beta$ then $\hat{P}:=\hat{P} \cup\left\{A_{i} \rightarrow \alpha A_{i} \beta\right\}$

end ;

return $\hat{G}$

end

Fig. 2. Procedure LG. 
then

$$
X \cap W_{k_{0}-1}(\alpha, \beta) \subset M_{W}(\alpha, \beta),
$$

On the other hand

$$
X \cap W_{k_{0^{-1}}}(\alpha, \beta) \subset X \cap M_{W}(\alpha, \beta)
$$

because

$$
X \cap W_{k_{0^{-1}}}(\alpha, \beta) \supset X \cap M_{W}(\alpha, \beta)
$$

$$
W_{k_{0}-1}(\alpha, \beta) \supset W_{\infty}(\alpha, \beta)=M_{W}(\alpha, \beta)
$$

Thus

$$
X \cap W_{k_{0}-1}(\alpha, \beta)=X \cap M_{W}(\alpha, \beta)=\hat{M}_{X ; W}(\alpha, \beta) .
$$

Since $W_{k}(\alpha, \beta) \subset W_{k_{0}-1}(\alpha, \beta)$ we have similarly

In Procedure DER,

$$
X \cap W_{k_{0}}(\alpha, \beta)=\hat{M}_{X ; W}(\alpha, \beta) .
$$

$$
Y=X \cap W_{k-1}(\alpha, \beta) \quad \text { and } \quad X^{\prime}=X \cap W_{k}(\alpha, \beta) .
$$

Input: Finite set $I_{+} \subset \Sigma^{*},\left(u_{0}, v_{0}\right),\left(\alpha_{0}, \beta_{0}\right) \in \Sigma^{*} \times \Sigma^{*}, n_{0} \in N$.

Output: Linear grammar $\hat{G}=\left(\hat{\Gamma}, \Sigma, \hat{P}, A_{1}\right)$.

procedure LG_INF $\left(I_{+},\left(u_{0}, v_{0}\right),\left(\alpha_{0}, \beta_{0}\right), n_{0}\right)$ :

begin

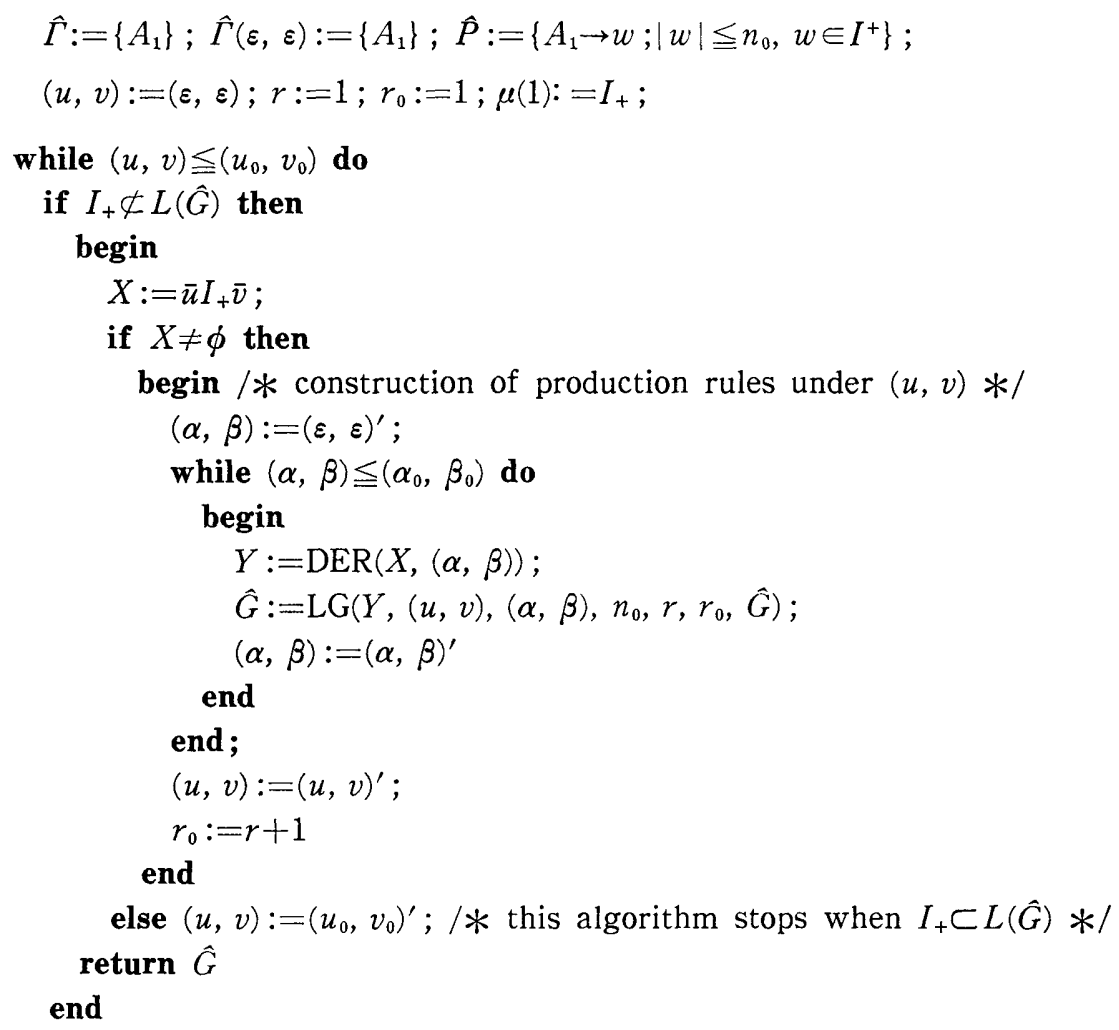

Fig. 3. Procedure LG_INF. 
Hence $Y \neq X^{\prime}$ if $k<k_{0} ; Y=X^{\prime}=\hat{M}_{X ; W}(\alpha, \beta)$ and Procedure DER stops if $k=k_{0}$. Therefore if $M_{W}(\alpha, \beta)=\phi$, then $Y=\hat{M}_{X ; W}(\alpha, \beta)=\phi$; if $M_{W}(\alpha, \beta) \neq \phi$, then $Y=\hat{M}_{X ; W}(\alpha, \beta) \supset X_{0}$ $\cap M_{W}(\alpha, \beta) \neq \phi$.

EXAMPLE 2. Let $W=\left\{a^{m} b^{m} ; m \geqq 0\right\} \cup\left\{a^{n} b^{2 n} ; n \geqq 0\right\}$ and $X=\{\varepsilon, a b, a b b\} \subset W$, then the output of Procedure DER is as follows:

(1) If $(\alpha, \beta)=(a, b)$, then

$$
Y=\{\varepsilon, a b\}=X \cap M_{W}(a, b) .
$$

(2) If $(\alpha, \beta)=(a, b b)$, then

$$
Y=\{\varepsilon, a b b\}=X \cap M_{W}(a, b b) .
$$

(3) Otherwise, $Y=\phi$.

\subsection{Construction of Production Rules}

The output of Procedure DER may be $Y=\hat{M}_{X ; W}(\alpha, \beta)$ for some input $X \subset W$. So our next interests is how to indicate new variables and construct new production rules from these outputs. If $M_{\bar{u} L \bar{v}}(\alpha, \beta)$ is nonempty and we can not find any old variable corresponding to it, then we produce a new variable and construct all production rules related to its variable. Such an Algorithm is given by Procedure LG in Fig. 2.

\begin{tabular}{|c|c|c|c|c|c|}
\hline$(u, v)$ & $(\alpha, \beta)$ & $Y$ & $\hat{\Gamma}$ & $\hat{P}$ & $L(\hat{G})$ \\
\hline$(\varepsilon, \varepsilon)$ & $(\varepsilon, \varepsilon)$ & $I_{+}$ & $A_{1}$ & $\phi$ & $\phi$ \\
\hline$(\varepsilon, \varepsilon)$ & $\begin{array}{l}(a, \varepsilon) \\
(\varepsilon, a) \\
(b, \varepsilon) \\
(\varepsilon, b)\end{array}$ & $\begin{array}{l}\phi \\
\phi \\
\phi \\
\phi\end{array}$ & & $\phi$ & $\phi$ \\
\hline$(a, \varepsilon)$ & $\begin{array}{l}(a, \varepsilon) \\
(\varepsilon, a) \\
(b, \varepsilon) \\
(\varepsilon, b)\end{array}$ & $\begin{array}{c}\phi \\
\phi \\
\{a a, b a b a\} \\
\phi\end{array}$ & $A_{2}$ & $A_{1} \rightarrow a A_{2}, A_{2} \rightarrow b A_{2}$ & $\phi$ \\
\hline$(\varepsilon, a)$ & $\begin{array}{l}(a, \varepsilon) \\
(\varepsilon, a) \\
(b, \varepsilon) \\
(\varepsilon, b)\end{array}$ & $\begin{array}{c}\phi \\
\phi \\
\phi \\
\{a a, a b a b\}\end{array}$ & $A_{3}$ & $A_{1} \rightarrow A_{3} a, A_{3} \rightarrow A_{3} b$ & $\phi$ \\
\hline$\dot{\cdot}$ & $\cdot$ & & & & \\
\hline$(a, a)$ & $\begin{array}{l}(a, \varepsilon) \\
(\varepsilon, a) \\
(b, \varepsilon) \\
(\varepsilon, b)\end{array}$ & $\begin{array}{c}\phi \\
\phi \\
\{a, b a b\} \\
\{a, b a b\}\end{array}$ & $A_{4}$ & $\begin{array}{l}A_{1} \rightarrow a A_{4} a, A_{4} \rightarrow b A_{4}, A_{4} \rightarrow a \\
A_{4} \rightarrow A_{4} b\end{array}$ & $L$ \\
\hline
\end{tabular}

Table 1. Inference process in Procedure LG_INF for $I_{+}=\{a a a, a b a b a\}$. 


\subsection{Inference of Linear Grammars}

In this subsection, we propose the algorithm to construct a linear grammar from a positive sample $I_{+}$of $L$ where $\left(u_{0}, v_{0}\right)$ is the upper limit on covers for generations of variables, $\left(\alpha_{0}, \beta_{0}\right)$ is the upper limit on covers for constructions of self-embedding rules and $n_{0}$ is the upper limit on lengths of right-side strings in the case of generating production rules of which the right-side consists of only terminals. Such an algorithm is given by Procedure LG_INF in Fig. 3 .

EXAMPLE 3. Let $L=\left\{a b^{m} a b^{n} a ; m, n \geqq 0\right\}, I_{+}=\{a a a, a b a b a\},\left(u_{0}, v_{0}\right)=(a, a),\left(\alpha_{0}, \beta_{0}\right)$ $=(\varepsilon, b)$ and $n_{0}=1$, then we can infer a linear grammar $\hat{G}=\left(\hat{\Gamma}, \Sigma, \hat{P}, A_{1}\right)$ such that $L=L(\hat{G})$ by using Procedure LG_INF as shown in Table 1 .

\section{Completeness for Linear Languages}

In this section we show that for any linear language $L, a$ grammar generating $L$ can be inferred by using Procedure LG_INF.

Lemma 6 . Let $L$ be an arbitrary language over $\Sigma$ and $G=(\Gamma, \Sigma, P,[L])$ be a linear grammar satisfying the following conditions. then $L(G) \subset L$ :

(1) $\Gamma$ is a set added the element $[L]$ to some finite subset of $\{[X]$; there is $(u, v) \in$ $\Sigma^{*} \times \Sigma^{*}$ such that $X \subset \bar{u} L \bar{v}$ and $\left.X \neq \phi\right\}$.

(2) $X_{2} \subset \bar{u} X_{1} \bar{v}$ if $\left(\left[X_{1}\right] \rightarrow u\left[X_{2}\right] v\right) \in P ; w \in X$ if $([X] \rightarrow w) \in P$.

PROOF. There is the following derivation for $x \in L(G)$ :

where

$$
\begin{aligned}
{\left[X_{0}\right] } & \Rightarrow u_{1}\left[X_{1}\right] v_{1} \Rightarrow u_{1} u_{2}\left[X_{2}\right] v_{2} v_{1} \Rightarrow \cdots \\
& \Rightarrow u_{1} \cdots u_{m} X_{m} v_{m} \cdots v_{1} \Rightarrow u_{1} \cdots u_{m} w v_{m} \cdots v_{1}=x
\end{aligned}
$$

$$
X_{0}=L, \quad\left(\left[X_{i}\right] \rightarrow u_{i+1}\left[X_{i+1}\right] v_{i+1}\right) \in P \quad(i=0, \cdots, m-1) .
$$

Since $\left(\left[X_{m}\right] \rightarrow w\right) \in P, w \in X_{m}$, that is, $x \in u_{1} \cdots u_{m} X_{m} v_{m} \cdots v_{1}$. Futhermore $X_{i+1} \subset$ $\bar{u}_{i+1} X_{i} \bar{v}_{i+1}$ by $\left(\left[X_{i}\right] \rightarrow u_{i+1}\left[X_{i+1}\right] v_{i+1}\right) \in P$, accordingly

Hence

$$
u_{i+1} X_{i+1} v_{i+1} \subset u_{i+1}\left(\bar{u}_{i+1} X_{i} \bar{v}_{i+1}\right) v_{i+1} \subset X_{i} \quad(i=0, \cdots, m-1)
$$

$$
x \in u_{1} \cdots u_{m} X_{m} v_{m} \cdots v_{1} \subset u_{1} \cdots u_{m-1} X_{m-1} v_{m-1} \cdots v_{1} \subset \cdots \subset u_{1} X_{1} v_{1} \subset L .
$$

LEMMA 7. In Procedure LG $I N F$, if $M_{L}(\alpha, \beta) \in \mathcal{E}_{\bar{u} L \bar{v}}$ and $\bar{u} I_{+} \bar{v} \neq \phi\left((u, v) \leqq\left(u_{0}, v\right)\right.$, $\left.(\alpha, \beta) \leqq\left(\alpha_{0}, \beta_{0}\right)\right)$, then there is an integer $m$ such that $\mu(m)=\hat{M}_{\bar{u} I_{+} \bar{v}}(\alpha, \beta)$.

PRoOF. Consider the case of $Y=\hat{M}_{\bar{u}_{I+} \tilde{v}}(\alpha, \beta)$. If there is $i(1 \leqq i \leqq r)$ such that $Y=$ $\mu(i)$, this lemma holds. Otherwise, we have $\mu(r+1)=Y=\hat{M}_{\bar{u} I_{+} \bar{v}}(\alpha, \beta)$.

Corollary. Let $G=(\Gamma, \Sigma, P, S)$ be a $C F G$ and $I_{+} \subset L(G)$. In Procedure $L G_{-} I N F$ under some $\left(u_{0}, v_{0}\right),\left(\alpha_{0}, \beta_{0}\right) \in \Sigma^{*} \times \Sigma^{*}$, for any variable $A \in \Gamma$ there is integer $m$ such that $\mu(m) \subset \bar{u} I_{+} \bar{v} \cap L_{A}$ and $A_{m} \in \hat{\Gamma}(u, v)$.

Proof. Given large enough $\left(u_{0}, v_{0}\right),\left(\alpha_{0}, \beta_{0}\right)$ there exist derivations such that $S \stackrel{*}{\Rightarrow} u A v$ and $A \stackrel{*}{\Rightarrow} \alpha A \beta\left((u, v) \leqq\left(u_{0}, v_{0}\right),(\alpha, \beta) \leqq\left(\alpha_{0}, \beta_{0}\right),(\alpha, \beta) \neq(\varepsilon, \varepsilon)\right)$. By Lemma $1, L_{A} \subset$ $M_{\bar{u} L \bar{v}}(\alpha, \beta)$. By Lemma 7 , there is an integer $m$ such that $\mu(m)=\bar{u} I_{+} \bar{v} \cap M_{\bar{u} L \bar{v}}(\alpha, \beta)$. Hence $\mu(m) \supset \bar{u} I_{+} \bar{v} \cap L_{A}$.

The above Corollary shows that each variable $A$ in CFG $G$ corresponds to a variable 
(written as $\hat{A}$ ) in the grammar $\hat{G}$ inferred from $I_{+} \subset L(G)$ using Procedure LG_INF. Futhermore, the following Lemma asserts that the language generated from $\hat{A}$ includes the language generated from $A$ if $G$ is linear:

LemMA 8. Let $G=(\Gamma, \Sigma, P, S)$ be a linear grammer and $L=L(G)$. Then for some $I_{+} \subset L, \quad\left(u_{0}, v_{0}\right), \quad\left(\alpha_{0}, \beta_{0}\right) \in \Sigma^{*} \times \Sigma^{*}$ and integer $n_{0}$, Procedure LG-INF infer the linear grammer $\hat{G}=\left(\hat{\Gamma}, \Sigma, \hat{P}, A_{1}\right)$ such that for any $A \in \Gamma$ there is $\hat{A} \in \hat{\Gamma}$ satisfying $L_{A} \subset L_{\hat{A}}$.

PROOF. Let

$$
\begin{aligned}
& \left(u_{0}, v_{0}\right)=\max \{(u, v) ; S \stackrel{*}{\Rightarrow} x \Rightarrow u y v \text { and each variable in } \Gamma \text { occurs at most once } \\
& \left.\quad \text { in the derivation process to } x \text { where } x, y \in(\Gamma \cup \Sigma)^{*}\right\}, \\
& \left(\alpha_{0}, \beta_{0}\right)=\max \{(\alpha, \beta) ;(A \rightarrow \alpha \mathrm{A} \beta) \in P, A \in \Gamma\}, \\
& n_{0}=\max \left\{|w| ;(A \rightarrow w) \in P, w \in \Sigma^{*}, A \in \Gamma\right\}, \\
& I_{+}=\left\{x ; x \leqq u_{0} \alpha_{0} \beta_{0} v_{0}, x \in L\right\} .
\end{aligned}
$$

Then for any $A \in \Gamma$, there exists a derivation such that

$$
S \stackrel{*}{\Rightarrow} u A v\left((u, v) \leqq\left(u_{0}, v_{0}\right)\right) \text {. }
$$

Now we consider the three forms of production rules from the variable

$$
A: \quad A \rightarrow \alpha A \beta, \quad A \rightarrow u^{\prime} B v^{\prime} \quad(A \neq B) \text { and } A \rightarrow w \quad\left(w \in \Sigma^{*}\right) .
$$

(1) the case of form $A \rightarrow \alpha A \beta$

$L_{A} \subset M_{\bar{u} L \bar{v}}(\alpha, \beta)$ by Lemma 1 and $\mu(m)=\hat{M}_{\bar{u} I_{+} \bar{v}}(\alpha, \beta)$ by Lemma 7 . Since $\mu(1)=I_{+}$, $\mu(1) \cap \hat{M}_{\bar{u} I_{+} \bar{v}}(\alpha, \beta) \neq \phi$. We now write $A_{m} \in \hat{\Gamma}$ as $\hat{A}$, then

$$
\left(A_{1} \rightarrow u \hat{A} v\right), \quad(\hat{A} \rightarrow \alpha \hat{A} \beta) \in \hat{P} .
$$

(2) the case of form $A \rightarrow u^{\prime} B v^{\prime}$

Consider the case of $Y=\hat{M}_{\overline{u u^{\prime}} I_{+} \overline{v^{\prime} v}}\left(\alpha^{\prime}, \beta^{\prime}\right)$, where

Then

$$
\left(B \rightarrow \alpha^{\prime} B \beta^{\prime}\right) \in P, \quad\left(u u^{\prime}, v^{\prime} v\right) \leqq\left(u_{0}, v_{0}\right) \quad \text { and } \quad\left(\alpha^{\prime}, \beta^{\prime}\right) \leqq\left(\alpha_{0}, \beta_{0}\right) .
$$

$$
\begin{aligned}
\bar{u}^{\prime} \mu(m) \bar{v}^{\prime} & =\bar{u}^{\prime}\left(\bar{u} I_{+} \bar{v} \cap M_{\bar{u} L \bar{v}}(\alpha, \beta)\right) \bar{v}^{\prime} \\
& =\overline{u u^{\prime}} I_{+} \overline{v^{\prime} v} \cap \bar{u}^{\prime} M_{\bar{u} L \bar{v}}(\alpha, \beta) \bar{v}^{\prime} .
\end{aligned}
$$

Futhermore, $Y \cap \vec{u}^{\prime} \mu(m) \bar{v}^{\prime} \neq \phi$ by Lemma 4. Thus

$$
\left(\hat{A} \rightarrow u^{\prime} \hat{B} v^{\prime}\right), \quad\left(\hat{B} \rightarrow \alpha^{\prime} \hat{B} \beta^{\prime}\right) \in \hat{P}
$$

where we write, as $\hat{B}, A_{r} \in \hat{\Gamma}$ in functional procedure LG.

(3) the case of form $A \rightarrow w$

Since we may assume that $|w| \leqq n_{0}$ and $w \in \mu(m), \quad(\hat{A} \rightarrow w) \in \hat{P}$. By the above analysis, we can understand that each production rule in $\hat{G}$ has its corresonding rule in $G$. Let $w$ be one of the minimal length words in $L_{A} \triangle L_{B}$ for $B \in \hat{\Gamma}$ distinct with $A$, such as $w \in L_{A}-L_{B}$, then there exist $\left(u_{1}, v_{1}\right),\left(u_{2}, v_{2}\right) \leqq\left(u_{0}, v_{0}\right)$ such that

Thus

$$
S \stackrel{*}{\Rightarrow} u_{1} A v_{1} \Rightarrow u_{1} w v_{1}, \quad S \stackrel{*}{\Rightarrow} u_{2} B v_{2} \nRightarrow u_{2} w v_{2} .
$$

$$
w \in \hat{M}_{\bar{u}_{1} I+\bar{v}_{1}}(\alpha, \beta)-\hat{M}_{\bar{u}_{2} I+\bar{v}_{2}}\left(\alpha^{\prime}, \beta^{\prime}\right)
$$


where $(A \rightarrow \alpha A \beta),\left(B \rightarrow \alpha^{\prime} B \beta^{\prime}\right) \in \hat{P}$. Namely $\hat{A} \neq \hat{B}$.

Innversely, $L_{A} \neq L_{B}$ if $\hat{A} \neq \hat{B}$, here $A \neq B$ by the predescrived restriction on grammar. Therefore $L_{A} \subset L_{\hat{A}}$ by application of the induction.

THEOREM 2. For any linear language there are some sample $I_{+} \subset L,\left(u_{0}, v_{0}\right),\left(\alpha_{0}, \beta_{0}\right)$ $\in \Sigma^{*} \times \Sigma^{*}$ and integer $n_{0}$ such that we can infer linear grammar $\hat{G}$ of $L$ by using Pro-

Input: $\quad x, y \in(\hat{\Gamma} \cup \Sigma)^{*}, \hat{G}=\left(\hat{\Gamma}, \Sigma, \hat{P}, A_{1}\right)$.

Output: Set of all strings composed from $x$ and $y ; H$.

Variables:

$m$ : Serial number of string analyzing currently.

$n$ : Maximal number of string in the middle of composition.

$z(m)$ : Composition string in the current.

procedure $\operatorname{COMPO}(x, y)$ :

begin

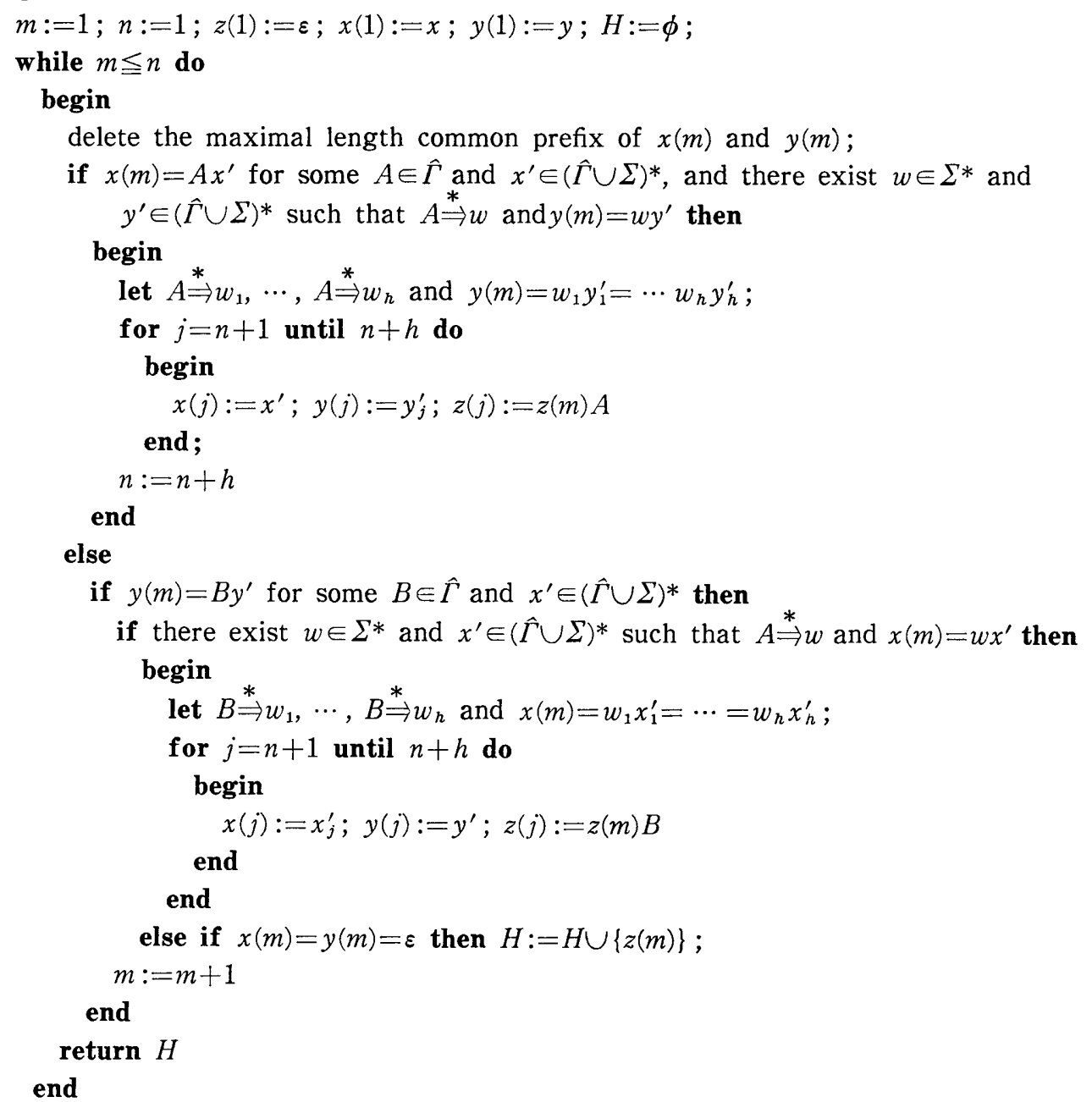

Fig. 4. Procedure COMPO. 
cedure $L G_{-} I N F$.

Proof. Taking that $A=S$ in Lemma $8, L \subset L(\hat{G})$. On the other hand, if we interpret $A_{r}$ obtained when $\mu(r)=\hat{M}_{\bar{u} I_{+} \bar{v}}(\alpha, \beta)$ in Procedure LG_INF, as $\left[M_{\bar{u} L \bar{v}}(\alpha, \beta)\right]$, then the inferred grammar $\hat{G}$ satisfies the condition of Lemma 6 and thus $L(\hat{G}) \subset L$.

Input: Finite set $I_{+} \subset \Sigma^{*},\left(u_{0}, v_{0}\right),\left(\alpha_{0}, \beta_{0}\right) \in \Sigma^{*} \times \Sigma^{*}, n_{0} \in N$.

Output: $\mathrm{CFG} \hat{G}=\left(\hat{\Gamma}, \Sigma, \hat{P}, A_{1}\right)$.

Variables:

check: 0 if it is impossible to compose the given strings for every variables;

1 otherwise.

$c(i)$ : $\quad 0$ if production rule from $A_{i}$ is not composed at all; 1 otherwise.

procedupe CFG_INF $\left(I_{+},\left(u_{0}, v_{0}\right),\left(\alpha_{0}, \beta_{0}\right), n_{0}\right)$;

\section{begin}

check : $=1$;

$\hat{G}:=\operatorname{LG}_{-} I N F\left(I_{+},\left(u_{0}, v_{0}\right),\left(\alpha_{0}, \beta_{0}\right), n_{0}\right)$;

for all $i(1 \leqq i \leqq r)$ do $c(i):=1$;

while $I_{+} \not \subset L(\hat{G})$ do

if $\operatorname{check} \neq 0$ then

begin

check $:=0$;

for $i=1$ until $r$ do

if $c(i)=1$ then

begin

check $:=1 ; c(i):=0$;

let $n(i)$ be the number of production rules from $A$ and $x_{1}, \cdots, x_{n(i)}$ be right-side strings of these rules;

for $p=1$ until $n(i)$ do

for $q=p$ until $n(i)$ do

if $\operatorname{COMPO}\left(x_{p}, x_{q}\right) \neq \phi$ then

begin

let $\operatorname{COMPO}\left(x_{p}, x_{q}\right)=\left\{y_{1}, \cdots, y_{t}\right\}$;

for $j=1$ until $t$ do

begin

$\hat{P}:=\hat{P} \cup\left\{A_{i} \rightarrow y_{j}\right\} ;$

if $I_{-} \cap L(\hat{G}) \neq \phi$ then $\hat{P}:=\hat{P}-\left\{A_{i} \rightarrow y_{j}\right\}$

else $c(i):=1$

end

end

end

end

else return $\phi$;

return $\hat{G} / * \hat{G}$ is compatible to given sample $\left(I_{+}, I_{-}\right) * /$

end

Fig. 5. Procedure CFG_INF. 
Hence $L=L(\hat{G})$.

\section{Grammatical Inference for CFL's}

We first give Procedure COMPO in Fig. 4 to compose production rules of linear grammars obtained by Procedure LG_INF. Then given a positive sample $I_{+} \subset L$ and a negative sample $I_{-} \subset \Sigma^{*}-L$ for a CFL $L$, we consider a method to adopt only composed rule compatible with these samples. In final, we propose Procedure CFG_INF to identify a CFG $G$ such that $L=L(G)$ in Fig. 5.

THEOREM 3. For any $C F L$, there are some sample $\left(I_{+}, I_{-}\right),\left(u_{0}, v_{0}\right),\left(\alpha_{0}, \beta_{0}\right) \in \Sigma^{*} \times \Sigma^{*}$ and integer $n_{0}$ such that we can infer a $C F G \hat{G}=\left(\hat{\Gamma}, \Sigma, \hat{P}, A_{1}\right)$ satisfying $L=L(\hat{G})$ in Procedure $C F G_{-} I N F$.

Proof. Let $L=L(G)$ and $G=(\Gamma, \Sigma, P, S)$. Let $A \rightarrow u_{0} A_{1} u_{1} \cdots u_{m-1} A_{m} u_{m}$ be a production rule of $G$, then by the similar method to Theorem 2 , there are $\left(\hat{A} \rightarrow u_{0} w_{1} u_{1} \cdots\right.$ $\left.u_{i-1} \hat{A}_{i} u_{i} \cdots w_{m} u_{m}\right)$ in $\hat{P}(i=1,2, \cdots, m)$ for some $I_{+} \subset L,\left(u_{0}, v_{0}\right),\left(\alpha_{0}, \beta_{0}\right) \in \Sigma^{*} \times \Sigma^{*}$ and $n_{0}$ $\in N$, where $w_{j}$ is a minimal length string in $L_{\boldsymbol{A}}(j=1, \cdots, m)$. By the composition method of rules in Procedure COMPO, we have $\left(\hat{A} \rightarrow u_{0} \hat{A}_{1} u_{1} \cdots u_{m-1} \hat{A}_{m} u_{m}\right) \in \hat{P}$. We can check whether these composed rules are compatible for $L$, by giving a large enough negative sample $I_{\text {-. }}$.

EXAMPLE 4. Let $L=\left\{a b^{m} a^{m} b a^{n} b^{n} a ; m, n \geqq 0\right\}$ be unknown. We apply Procedure CFG_INF for $I_{+}=\{a b a, a b a b a, a b a b a b a\} \subset L, I_{-}=\{a b a a b b a b a\} \not \subset L, \quad\left(u_{0}, v_{0}\right)=(a, b a)$. $\left(\alpha_{0}, \beta_{0}\right)=(b, a)$ and $n_{0}=0$. First, by the function procedure LG_INF, a linear grammar $\hat{G}=\left(\left\{A_{1}, A_{2}, A_{3}\right\},\{a, b\}, \hat{P}, A_{1}\right)$ is obtained as shown in Table 2 , where $\hat{P}=\left\{A_{1} \rightarrow\right.$ $\left.a b A_{2} a / a A_{3} b a, A_{2} \rightarrow a A_{2} b / \varepsilon, A_{3} \rightarrow b A_{3} a / \varepsilon\right\}$.

Composing these production rules in ordering from $A_{1}$,

(1) $\operatorname{COMPO}\left(a b A_{2} a, a b A_{2} a\right)=\left\{a b A_{2} A_{2} a\right\}$ :

If we add $\left(A_{1} \rightarrow a b A_{2} A_{2} a\right)$ to $\hat{P}$, then it derives a contradiction because $a b a a b b a b a$

Table 2. Inference process in Procedure CFG_INF for $I_{+}=\{a b a, a b a b a, a b a b a b a\}$.

\begin{tabular}{|c|c|c|c|c|c|}
\hline$(u, v)$ & $(\alpha, \beta)$ & $Y$ & $\hat{\Gamma}$ & $\hat{P}$ & $L(\hat{G})$ \\
\hline$(\varepsilon, \varepsilon)$ & $(\varepsilon, \varepsilon)$ & $I_{+}$ & $A_{1}$ & $\phi$ & $\phi$ \\
\hline • & - & & & & \\
\hline$(a b, a)$ & $\begin{array}{c}\cdot \\
\cdot \\
(a, b) \\
\cdot \\
\cdot\end{array}$ & $\{\varepsilon, a b\}$ & $A_{2}$ & $A_{1} \rightarrow a b A_{2} a, A_{2} \rightarrow a A_{2} b, A_{2} \rightarrow \varepsilon$ & $\begin{array}{l}a b a b a b a \\
\notin L(\hat{G})\end{array}$ \\
\hline$(a, b a)$ & $\begin{array}{c}\cdot \\
(b, a)\end{array}$ & $\{\varepsilon, b a\}$ & $A_{3}$ & $A_{1} \rightarrow a A_{3} b a, A_{3} \rightarrow b A_{3} a, A_{3} \rightarrow \varepsilon$ & $\begin{array}{l}a b a b a b a \\
\notin L(\hat{G})\end{array}$ \\
\hline
\end{tabular}


$\in I_{-}$is generated only by applying this rule.

(2) $\operatorname{COMPO}\left(a b A_{2} a, a A_{3} b a\right)=\left\{a A_{3} b A_{2} a\right\}$ :

If we add $\left(A \rightarrow a A_{3} b A_{2} a\right)$ to $\hat{P}$, then Procedure CFG_INF terminates because $I_{+} \subset$ $L(\hat{G})$ and $I_{-} \cap L(\hat{G})=\phi$. Indeed, $L=L(\hat{G})$.

\section{References}

[1] Soromonoff, R. : A Formal Theory of Inductive Inference, Information and Control, (1964), $1-22,224-254$.

[2] Huzino, S.: On Some Properties of Derivative-mappings, Structural Diagrams and Structural Equations: Part 1, Memo. Fac. Sci. Kyushu Univ. Ser. A20, (1966), 179-265.

[3] GolD, M.: Language Identification in the Limit, Information and Control, 10 (1967), $447-474$

[4] Feldman, J A., Gips, J., Horning, J. J. and Reader, S.: Grammatical Complexity and Inference, Technical Report No. CS125, Computer Science Department, Stanford University (1969).

[5] Biermann, A. W.: An Interactive Finite-state Language Learner, Proc. 1-st USA-JAPAN Comp. Conf. (1972), 13-20.

[6] Tanatsugu, K. and Arikawa, S.: On Characteristic Sets and Degrees of Finite Automata, International Journal of Computer and Information Sciences, 6, 1 (1977), 83-93.

[7] Tanatsugu, K.: A Grammatical Inference for Harmonic Linear Languages, International Journal of Computer and Information Sciences, 13, 5 (1984), 413-423.

Communicated by S. Kan̄̄

Received October 14,1986

Revised October 30, 1986 